THURSDAY, 04 JUNE 2020 Rheumatoid arthritis - aetiology, pathogenesis and
animal models

\section{THU0063 DIFFERENTIAL PHARMACODYNAMIC EFFECTS OF ABATACEPT AND ADALIMUMAB ON THE SERUM PROTEOME OF PATIENTS WITH RA USING THE SOMASCAN@ PLATFORM}

D. Galbraith ${ }^{1}$, M. Caliskan ${ }^{1}$, O. Jabado' ${ }^{1}$ S. Hu ${ }^{1}$, R. Fleischmann ${ }^{2}$, M. Weinblatt ${ }^{3}$,

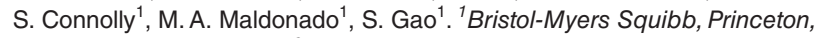
United States of America; ${ }^{2}$ University of Texas Southwestern Medical Center, Dallas, United States of America; ${ }^{3}$ Brigham and Women's Hospital, Boston, United States of America

Background: Abatacept (ABA) versus adaliMumab (ADA) comParison in bioLogic-naïvE RA subjects with background MTX (AMPLE) was a Phase Illb clinical trial to compare the safety, efficacy and radiographic outcomes of $A B A$ vs ADA in patients with RA who exhibited an inadequate response to MTX and who were naïve to biologic DMARDs. ${ }^{1}$ While both therapies demonstrated similar efficacy across multiple outcomes, their mechanisms of action (MoAs) are quite different; ABA is a T-cell co-stimulation modulator and ADA is a TNFa inhibitor. Previous transcriptomic analysis of the whole blood samples showed differential pharmacodynamic (PD) effects between the treatments. ${ }^{1-3}$

Objectives: To expand our understanding of differential PD changes in the serum proteome over time in patients treated with ABA or ADA in AMPLE using a novel proteomic platform.

Methods: Serum was available from 440 patients in AMPLE at four time points (Days 1, 85, 365 and 729). Serum samples from the patients in AMPLE and 123 healthy individuals with matching demographics were subjected to proteomic quantification by a highly multiplexed DNA aptamer technology with wide dynamic ranges (SomaLogic SomaScan ${ }^{\circledR}$ platform).${ }^{4} \mathrm{~A}$ linear model analysis was used to identify protein abundance changes over time and changes specific to treatment. Other covariates included in the model were country of origin, ethnicity and sex. Additionally, patient effect was adjusted for as a random factor.

Results: Both treatments exhibited a significant PD effect on serum proteome over the course of the 2-year trial, with 73 proteins modulated by ABA and 125 by ADA. There were large overlaps between the two treatments, including proteins associated with RA, such as C-X-C motif chemokine ligand 13 (CXCL13), matrix metalloproteinase-3 (MMP3) and serum amyloid A1/A2 (SAA1/2). Changes in the levels of these proteins may be indicative of general improvement of the disease. The proteins modulated by the treatments were enriched in the G-protein coupled receptor (GPCR) signalling and innate immunity pathways. Among the proteins that exhibited significantly different PD effects between the treatments were CRP, CC chemokine ligand 17 (CCL17) and $\beta$-defensin 112 (Figure). While patients showed marked improvement in their symptoms after 2 years of treatment, the overall serum proteomic profiles of the patients were still different from those of a normal healthy population.

Conclusion: The SomaScan ${ }^{\circledast}$ platform provides a robust method for quantifying the PD change in a broad portion of the serum proteome in clinical trials. In AMPLE, abatacept was more selective than adalimumab in modulating protein biomarkers in patients with RA, though there was large overlap in proteins modulated by both treatments. The treatment-specific changes may reflect the different MoAs leading to similar clinical outcomes. While patients in both groups benefited from treatments, their serum proteome remained notably different from that of a healthy population. Further analysis by responder status may provide additional links between the treatment responses and proteomic changes. Proteomic approaches as described in our study could contribute to clinical trials and help shape treatment strategies for patients with RA.

References:

[1] Schiff M, et al. Ann Rheum Dis 2014;73:86-94.

[2] Bandyopadhyay S, et al. Arthritis Rheum 2014;66:Abstract 1520.

[3] Sokolove J, et al. Ann Rheum Dis 2016;75:709-14.

[4] Gold L, et al. PLoS One 2010;5:e15004.

Disclosure of Interests: David Galbraith Shareholder of: Bristol-Myers Squibb, Employee of: Bristol-Myers Squibb, Minal Caliskan Employee of: Bristol-Myers Squibb, Omar Jabado Shareholder of: Bristol-Myers Squibb, Employee of: Bristol-Myers Squibb, Sarah Hu Shareholder of: Bristol-Myers Squibb, Employee of: Bristol-Myers Squibb, Roy Fleischmann Grant/research support from: AbbVie, Akros, Amgen, AstraZeneca, Bristol-Myers Squibb, Boehringer, IngelhCentrexion, Eli Lilly, EMD Serono, Genentech, Gilead, Janssen, Merck, Nektar, Novartis, Pfizer, Regeneron Pharmaceuticals, Inc., Roche, Samsung, Sandoz, Sanofi Genzyme, Selecta, Taiho, UCB, Consultant of: AbbVie, ACEA, Amgen, Bristol-Myers Squibb, Eli Lilly, Gilead, GlaxoSmithKline, Novartis, Pfizer, Sanofi

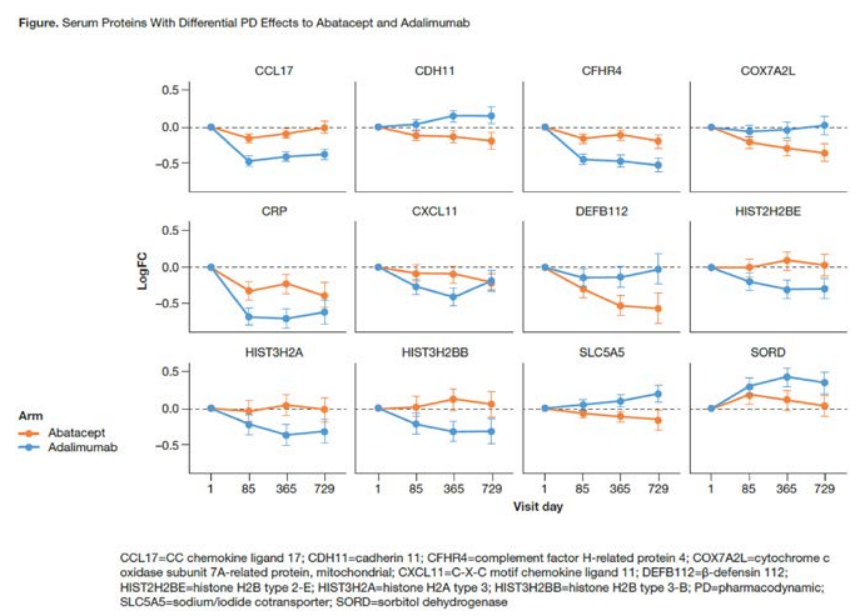

Genzyme, UCB, Michael Weinblatt Grant/research support from: Amgen, Bristol-Myers Squibb, Crescendo, Lily, Sanofi/Regeneron, Consultant of: AbbVie, Amgen, Bristol-Myers Squibb, Crescendo, Gilead, Horizon, Lily, Pfizer, Roche, Sean Connolly Shareholder of: Bristol-Myers Squibb, Employee of: Bristol-Myers Squibb, Michael A Maldonado Shareholder of: Bristol-Myers Squibb, Employee of: Bristol-Myers Squibb, Sheng Gao Shareholder of: Bristol-Myers Squibb, Employee of: Bristol-Myers Squibb

DOI: 10.1136/annrheumdis-2020-eular. 1452

\section{THU0064 \\ ANTIBODY-RESPONSE MATURATION IN THE PHASE OF CLINICALLY SUSPECT ARTHRALGIA AND ITS RELATION WITH PROGRESSION TO RHEUMATOID ARTHRITIS}

F. Wouters ${ }^{1}$, E. Niemantsverdriet ${ }^{1}$, N. Salioska ${ }^{1}$, A. L. Dorjée ${ }^{1}$, R. Toes ${ }^{1}$, A. Van der Helm - van Mil ${ }^{1,2}$. ${ }^{1}$ Leiden University Medical Center, Department of Rheumatology, Leiden, Netherlands; ${ }^{2}$ Erasmus Medical Center, Department of Rheumatology, Rotterdam, Netherlands

Background: Auto-antibodies in rheumatoid arthritis (RA) are often present years before disease onset but their mere presence does not seem enough to induce RA. Because several nested-case control studies have shown that autoantibody-response maturation precedes disease onset, it is suggested that it plays a role in disease triggering. At present, it is undetermined whether autoantibody-response maturation occurs in the symptomatic phase preceding clinical arthritis (i.e. Clinically Suspect Arthralgia, CSA), or whether it occurs even earlier in the asymptomatic phase. Secondly, if autoantibody-response maturation is a final step towards clinical disease development, maturation is expected to be present in the patients that progress from CSA to RA, but not in CSA-patients that do not progress.

Objectives: To better understand the timeframe of autoantibody-response maturation and its relation to development of RA, we investigated autoantibody-response maturation in patients with CSA that did and did not progress to clinically apparent inflammatory arthritis (IA).

Methods: In serum from 148 CSA-patients, we determined the presence and levels of three autoantibodies (ACPA, anti-CarP and AAPA), with three isotypes each $(\operatorname{lgM}, \lg G, \lg A)$, resulting in 9 autoantibody measurements per patient per time-point. Measurements were performed on sera obtained at first presentation at the outpatient clinic and when patients developed IA or else after two years. In-house ELISA was used for all measurements. Three analyses were performed, in patients that progressed to IA $(n=56)$ and in patients that did not progress $(n=92)$ separately. First, in patients negative for all measurements at baseline, we determined the frequency of conversion to seropositivity. Second, in patients with at least one positive test at baseline, we studied the frequency of autoantibody positivity over time. Finally, we determined the change in autoantibody levels in patients positive for the respective autoantibodies at baseline. Frequencies and medians were reported. Statistical significance was tested with Fisher's Exact test and GEE, taking into account that measurements within one autoantibody type (ACPA, anti-CarP or AAPA) can be correlated.

Results: First we studied patients negative for all antibodies at baseline $(54 \%$ of patients that progressed to IA and $76 \%$ of patients that did not progress). $17 \%$ of patients that progressed to IA became positive over time, compared to $6 \%$ of the patients that did not develop IA ( $p=0.12)$. Then we studied patients in whom at least one autoantibody was present at baseline and evaluated autoantibody-positivity over time. In patients that progressed to IA, the number 
of autoantibodies detected at baseline did not change significantly during follow-up (median 1.5 (IQR 1-3, max. 6) to 1.0 (IQR 1-4, max. 6) $(p=0.18)$ ). In the patients with CSA that did not progress similar findings were made $(1.0$ (IQR 1-2, max. 4) at baseline and 1.0 (IQR 0-2, max. 5) after 2-years ( $p=0.07)$ ). Increase in number of positive measurements over time took place in only few patients: in $15 \%$ of the patients that progressed to IA and in $18 \%$ from those that did not progress $(p=1.00)$. Likewise, autoantibody levels did not significantly change over time, both in patients that progressed and in patients that did not progress.

Conclusion: The presence of IgM, IgG and IgA ACPA, anti-CarP and AAPA, as well as autoantibody levels, did not significantly increase over time in patients with CSA; this was similar for patients that did and did not develop clinical arthritis. These findings suggest that antibody-response maturation occurs before presenting with symptoms and also that broadening of the autoantibody response is not specific for progression from arthralgia to RA.

Disclosure of Interests: None declared

DOI: 10.1136/annrheumdis-2020-eular.3217

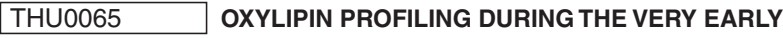 PHASE OF RHEUMATOID ARTHRITIS: ASSOCIATIONS WITH DISEASE STAGE, CLINICAL FEATURES AND TREATMENT RESPONSE}

J. Rodríguez-Carrio ${ }^{1,2,3}$, R. Coras $^{4}$, M. Alperi-López ${ }^{5}$, P. López ${ }^{1,2}$, F. J. BallinaGarcía $^{5}$, M. Guma ${ }^{4}$, A. Suárez ${ }^{1,2} .{ }^{1}$ University of Oviedo, Area of Immunology, Oviedo, Spain; ${ }^{2}$ Instituto de Investigación Sanitaria del Principado de Asturias, Oviedo, Spain; ${ }^{3}$ Hospital Universitario Central de Asturias, Bone and Mineral Research Unit, REDinREN, Oviedo, Spain; ${ }^{4}$ UCSD, Department of Medicine, San Diego, United States of America; ${ }^{5}$ Hospital Universitario Central de Asturias, Oviedo, Spain

Background: traditional approaches and lipidomics support the relevance of eicosanoids in rheumatic conditions, such as rheumatoid arthritis (RA). Previous studies from our group and others have revealed altered levels of arachidonic acid in RA, pointing to a possible consumption or enhanced metabolisation. However, the potential alterations of its actual metabolites are difficult to assess with conventional approaches, and new, untargeted, high-throughput technologies are needed. More importantly, whether these alterations are related to the disease course and could be found at the very early stage of the disease is unknown.

Objectives: to characterize the eicosanoid profiles during the earliest stages of RA and their potential clinical associations.

Methods: 60 very early RA patients (50 recruited at diagnosis and treatment-naïve) fulfilling 2010 ACR/EULAR criteria, 11 clinical suspect arthralgia (CSA) individuals and 28 healthy controls $(\mathrm{HC})$ were recruited. Samples were collected at the moment of the diagnosis. Serum oxylipins profiles were analyzed by mass spectrometry (LC-MS/MS). Treatment-naïve patients underwent csDMARD treatment and were followed for $6(n=49)$ and 12 months $(n=38)$. Data analysis was performed in $\mathrm{R}$ and MetaboAnalyst.

Results: A total of 75 oxylipins, mostly derived from arachidonic (AA), eicosapentanoic (EPA) and linoleic (LA) acid, were identified. No effect was observed for age, gender or BMI. Correlation and network analyses revealed different patterns among oxylipins across RA patients, CSA and HC (Figure 1A). The 8-HETrE, PGE3 and 20-HETE showed the pattern (linear increase) $\mathrm{HC} \rightarrow \mathrm{CSA} \rightarrow \mathrm{RA} \quad(\mathrm{p}=1.47 \cdot 10-4,5.34 \cdot 10-4$ and $5.68 \cdot 10-4$, respectively; and adjusted FDR<0.050) (Figure 1B). A PLS-DA (explaining $12.3 \%$ of the total variance, with a $71.0 \%$ cross-validation accuracy and permutation $p=5 \cdot 10-4$ ) confirmed that oxylipins profiles differ among groups, although a certain overlap existed. A total of 22 oxylipins had VIP scores $>1$ (Figure 1C), which allowed the identification of two clusters (I and II). Cluster usage (I/II) differed among groups $(p=0.003)$ : HC (27/1), CSA (7/4) and RA (37/23). Patients exhibiting cluster II showed higher VAS global assessment $(p=0.016)$ and pain $(p=0.003)$ than their cluster I-counterparts. More importantly, cluster II patients were less likely to achieve DAS28 remission at $6(12 / 17$ vs $10 / 32, p=0.008)$ and 12 months $(6 / 9$ vs $9 / 29, p=0.066)$ upon conventional DMARD treatment compared to those showing cluster $\mathrm{I}$.

OPLS-DA analyses revealed a good discrimination between CSA and $\mathrm{HC}$ groups, and 7 compounds (13-HODE, PGB2, 9-oxo-ODE, 12-oxo-ETE, 19,20-di-HDPA, 5-HETrE and 15-HEPE) were associated with the course HC->CSA. Different precursors (2 LA, 3 AAA, 1 EPA and $1 \mathrm{DHA}$ ) and pathways were noted ( 3 LOX, 4 CYP450). Regarding RA subsets, differences were noted by seropositivity. Whereas 9 compounds were associated with the pattern $\mathrm{HC} \rightarrow$ seronegative $\mathrm{RA}$ (8-HETrE, PGE3, 20-HETE, 19,20-di-HDPA, PGEM, PGJ2, 12-oxo-LTB4, 14,15EET and LTB4), a distinct set was observed for the pattern $\mathrm{HC} \rightarrow$ seropositive RA (PGE3, 20-oh-PGE2, 5-HETE, PGE2, 12-oxo-ETE, 20-HETE, PGEM, 4-HDoHE, LTB4, 9-oxo-ODE, 12-oxo-LTB4, 8,9-EET). No differences in the major pathways were noted.
Conclusion: Oxylipin networks differ across disease stages during the very early phase of RA, and can inform on specific signatures related to the disease progression. Oxylipins can delineate profiles with clinical relevance and are able to predict treatment response.

Figure:

(A)
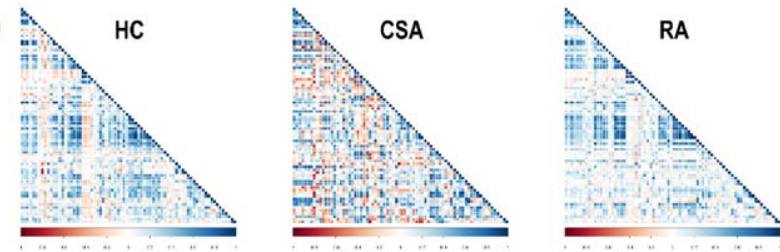

(B)

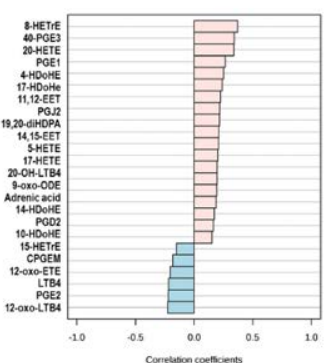

(C)

Disclosure of Interests: None declared

DOI: 10.1136/annrheumdis-2020-eular.3236

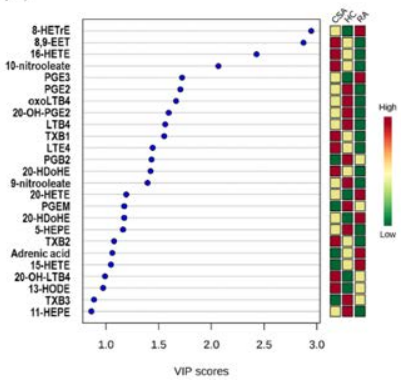

\begin{tabular}{l|l}
\hline THU0066 & DOES PORPHYROMONAS GINGIVALLIS \\
MODULATE GUT MICROBIOME RESULTING \\
IN AGGRAVATION OF DISEASE ACTIVITY IN \\
RHEUMATOID ARTHRITIS?
\end{tabular}

K. Kitamura ${ }^{1}$, K. Terato ${ }^{2}$, R. Fukai ${ }^{3}$, K. Katayama ${ }^{4}$, T. Waritani ${ }^{2}$, H. Shionoya ${ }^{1}$.

${ }^{1}$ Asama Chemicals, Tokyo, Japan; ${ }^{2}$ Chondrex Inc, Redmond, United States of America; ${ }^{3}$ Fukai Pharmacy, Asahikawa, Japan; ${ }^{4}$ Katayama Orthopedic Rheumatology Clinic, Asahikawa, Japan

Background: Oral Porphyromonas gingivalis $(\mathrm{Pg})$ infection in rheumatoid arthritis (RA) model revealed aggravation of arthritis, dysbiosis and higher serum LPS (1). We reported that $\lg A / \lg G$ antibody against Pg-LPS ratio among the patients with RA related to disease marker levels and disease activities (2). However, there are still few reports related to Gut-Oral axis.

Objectives: Firstly, how total intestinal bacteria, well-known 5 species of gut bacteria behave in relation to disease activities and markers. Secondly, how infection by $\mathrm{Pg}$ aggravate $\mathrm{RA}$ in relation to microflora, disease activities and markers.

Methods: Eighty-seven RA patients with inadequate response to conventional synthetic DMARDs were evaluated. Age: $68.1 \pm 8.7$ years, female (\%): 79.3 , dis ease duration: $136 \pm 91$ months, DAS28-ESR: $4.68 \pm 0.98$. Fecal bacteria num bers of total bacteria, Bifidobacterium, Lactobacillus, E. coli, Bacteroides and Staphylococcus were determined by PCR analysis. Fecal and serum LPS levels were measured by LAL assay. Serum endotoxin neutralizing activity (ENC) was determined along with RA disease activity and markers measurement. Infection of $\mathrm{Pg}$ was proofed by $\lg \mathrm{G}$ and $\operatorname{lgA}$ anti-LPS antibody measurement. The statistical relationships between variables were analyzed by Spearman's non-parametric rank correlation analysis and expressed as Spearman's rank correlation coefficient " $\rho$ ".

Results: At first, we could neglect background effects as duration, methotrexate dose for measuring total or each bacterial number (data not shown). As shown in Figure 1, total bacteria only showed positive correlation with IL-6 levels ( $\rho=$ $0.230, p=0.034$ ), however inversely correlated with disease activities such as pVAS $(\rho=-0.223, p=0.038)$ and Pain VAS $(\rho=-0.260, p=0.015)$. While no significant relationship was observed between the numbers of each of 5 species of bacteria and the disease activities and makers except Bifidobacterium. In contrast, LPS-related markers showed positive correlation with disease activities and markers. Among LPS-related biomarker values, serum LBP levels mainly correlated with most of RA biomarker-ESR $(\rho=0.497, p<0.0001)$, CRP $(\rho=$ $0.697, p<0.0001)$, MMP3 ( $\rho=0.546, p<0.0001$ ) and so on. Fecal LPS level was shown significant positive correlation with disease activities, however almost no correlation was observed in serum LPS level. IgA anti-LPS antibody levels to $\mathrm{Pg}$, resulted in inverse correlation with total bacteria $(\rho=-0.441, p<0.0001)$, Lactobacillus $(\rho=-0.224, p=0.037)$, Bacteroides $(\rho=-0.200, p=0.064)$ and $E$. coli 